\title{
Improved Error Estimates for Numerical Solutions of Symmetric Integral Equations
}

\author{
By E. Rakotch
}

Abstract. The most widely employed method for a numerical sclution of a symmetric integral equation with kernel $K(x, t)$ in interval $I \equiv[a, b]$ is the replacement of the original problem by the sequence of eigenproblems

$$
K^{(n)} y_{i}^{(n)}=\mu_{i n} y_{i}^{(n)}, \quad K^{(n)} \equiv\left\{w_{j n} K\left(x_{i n}, x_{j n}\right)\right\}, \quad i=1, \ldots, n,
$$

with $w_{j n}>0$ and $x_{j n} \in I, j=1, \ldots, n$. The eigenvectors $y_{i}^{(n)}$ are further used to obtain an approximation, with improved error estimates, of the numerical eigensolution for some $N>n$, with no necessity of computing $\mu_{i N}$ and $y_{i}^{(N)}, i=1, \ldots, N$, and of constructing another matrix.

1. Introduction. Let $K(x, t)$ be a Hermitian kernel defined in $I \times I$, where $I \equiv$ $[a, b]$, i.e. $K(t, x)=\overline{K(x, t)}$, such that

$$
F(x) \equiv \int_{a}^{b}|K(x, t)|^{2} d t \quad \text { is bounded in } I .
$$

It is known that all the characteristic values $\mu_{i}$ of $K(x, t)$ are real and there exists an orthonormal set $\left\{y_{i}(x)\right\}$ of characteristic functions [2], i.e.

$$
\int_{a}^{b} K(x, t) y_{i}(t) d t=\mu_{i} y_{i}(x), \quad \int_{a}^{b} y_{i}(x) \overline{y_{j}(x)} d x=\delta_{i j} .
$$

The first attempt to obtain a numerical solution for (1) with an error estimate for the characteristic values was made by Wielandt [4], which replaced the original problem by the sequence of eigenproblems

$$
K^{(n)} y_{i}^{(n)}=\mu_{i n} y_{i}^{(n)}, \quad K^{(n)} \equiv\left\{w_{j n} K\left(x_{i n}, x_{j n}\right)\right\}, \quad i=1, \ldots, n ;
$$

$w_{j n}>0$ and $x_{j n} \in I, j=1, \ldots, n$, are, respectively, the weights and the nodes of an integration rule $S$ by which the approximation

$$
\int_{a}^{b} f(x) d x \simeq \sum_{i=1}^{n} w_{i n} f\left(x_{i n}\right)
$$

is made. The eigenvalues $\mu_{k n}, k=1, \ldots, n$, which are all real, are then taken by Wielandt as approximations to the corresponding characteristic values of $K(x, t)$, where the correspondence is specified by the following assumptions:

Let $V \equiv\left\{\alpha_{1}, \ldots, \alpha_{m}\right\}$ be a subset of the set $R$ of all eigenvalues of a square matrix $A$ or of all characteristic values of a kernel $F(x, t)$ defined in $I \times I$, and let $W \equiv\left\{z^{2} \mid z \in V\right\} ;$ then,

(a) if $\alpha_{1}, \ldots, \alpha_{m}$ are the $m$ largest (smallest) real elements of $R$ such that 
$\alpha_{1} \geqslant \alpha_{2} \geqslant \cdots \geqslant \alpha_{m}\left(\alpha_{1} \leqslant \alpha_{2} \leqslant \cdots \leqslant \alpha_{m}\right)$, then every $\alpha_{i} \neq \alpha_{m}$ with multiplicity $r_{i} \geqslant 1$ occurs $r_{i}$ times in $V$,

(b) if $\alpha_{1}, \ldots, \alpha_{m}$ are the $m$ real elements of $R$ of the largest modulus such that $\left|\alpha_{1}\right| \geqslant\left|\alpha_{2}\right| \geqslant \cdots \geqslant\left|\alpha_{m}\right|$ and there are $r_{i}$ real elements of $R$ of modulus $\left|\alpha_{i}\right|$, then every $\alpha_{i}^{2} \neq \alpha_{m}^{2}$ occurs $r_{i}$ times in $W$.

Wielandt obtained the error estimates and the convergence properties for the eigenvalues with integration rules $S$ such that

$$
\eta_{n}(x, t) \equiv \sum_{i=1}^{n} w_{i n} K\left(x, x_{i n}\right) K\left(x_{i n}, t\right)-\int_{a}^{b} K(x, z) K(z, t) d z
$$

converges to 0 , as $n \rightarrow \infty$, uniformly in $I \times I$, and they were further improved and extended to every integration rule satisfying (3) [3], together with an error estimate for the numerical solutions generated by the eigenvectors of (2), as defined in [3, Section 3]. The purpose of this paper is to obtain new numerical solutions for (1) with improved error estimates for those ones corresponding to simple characteristic values in terms of the error estimates, obtainable either by [4] or [3], for the eigensolution of (2) with $n$ replaced by some $N>n$. This approach is due originally to $\operatorname{Linz}[1]$.

2. Error Estimation. The following theorem is first applied for the new error estimation [5, pp. 139-140]:

TheOREM 1. Let $A$ be a Hermitian matrix of order $m$ with eigenvalues $\lambda_{1}, \lambda_{2}$, $\ldots, \lambda_{m}$ and $y \equiv\left(y_{1}, y_{2}, \ldots, y_{m}\right) \in C_{m}$-the m-dimensional complex Euclidean space-with

$$
|y| \equiv\left[\sum_{i=1}^{m}\left|y_{i}\right|^{2}\right]^{1 / 2}=1
$$

then for every number $\mu$

$$
\min _{i}\left|\mu-\lambda_{i}\right| \leqslant|A y-\mu y| \text {. }
$$

The smallest value of $|A y-\mu y|$ with $|y|=1$ is attained for $\mu=(A y, y)$, which by Theorem 1 yields:

Theorem 2. Let $A$ be a Hermitian matrix of order $m$ with eigenvalues $\lambda_{1}, \lambda_{2}$, $\ldots, \lambda_{m}$; then for every $y \in C_{m}$ such that $|y|=1$ and for every $\mu$

$$
\min _{i}\left|(A y, y)-\lambda_{i}\right| \leqslant|A y-\mu y| \text {. }
$$

Define now a new scalar product $(u, v)_{m}$ of $u, v \in C_{m}$ and a new norm $|u|_{m}$ by

$$
(u, v)_{m} \equiv \sum_{i=1}^{m} w_{i m} u_{i} \bar{v}_{i}, \quad|u|_{m} \equiv \sqrt{(u, u)_{m}} .
$$

To obtain a numerical solution for a characteristic function, the eigenvectors $y_{k}^{(n)}$ of (2) were assumed to satisfy $\left|y_{k}^{(n)}\right|_{n}=1$ [3, Section 3]. Now, given an approximate eigensolution $\tilde{\mu}_{k n}, \tilde{y}_{k}^{(n)}$ of (2), the numerical solution $\tilde{y}_{k n}(x)$ for a characteristic function generated by $\widetilde{y}_{k}^{(n)}$ can be defined, as in [3, Section 3], by 


$$
\tilde{y}_{k n}(x) \equiv \tilde{\mu}_{k n}^{-1} \sum_{j=1}^{n} w_{j n} \tilde{y}_{k j}^{(n)} K\left(x, x_{j n}\right) .
$$

Further, take $N>n$ and, using (4), obtain approximations $\widetilde{y}_{k}^{(N)}$ for $y_{k}^{(N)}$ and $\widetilde{\mu}_{k N}$ for $\mu_{k N}$ and a numerical solution $\tilde{y}_{k N}(x)$ for the characteristic function as

$$
\begin{aligned}
\tilde{y}_{k}^{(N)} & \equiv\left|Y_{k}^{(N)}\right|_{N}^{-1} Y_{k}^{(N)}, \text { where } Y_{k i}^{(N)} \equiv \tilde{y}_{k n}\left(x_{i N}\right), i=1, \ldots, N, \\
\tilde{\mu}_{k N} & \equiv\left(K^{(N)} \tilde{y}_{k}^{(N)}, \tilde{y}_{k}^{(N)}\right)_{N}, \\
\tilde{y}_{k N}(x) & \equiv \tilde{\mu}_{k N}^{-1} \sum_{j=1}^{N} w_{j N} \tilde{y}_{k j}^{(N)} K\left(x, x_{j N}\right) ;
\end{aligned}
$$

then for a simple characteristic value $\mu_{k}$ it can be shown that the error estimate for $\widetilde{\mu}_{k N}$ is of better order of magnitude than that obtained for $\mu_{k n}$ by [3, Theorem 1], provided that $\left|\widetilde{y}_{k}^{(n)}-y_{k}^{(n)}\right|_{n}$ and $\left|\widetilde{\mu}_{k n}-\mu_{k n}\right|$ are sufficiently small. Indeed,

$$
\left|\tilde{\mu}_{k N}-\mu_{k}\right| \leqslant\left|\tilde{\mu}_{k N}-\mu_{k N}\right|+\left|\mu_{k N}-\mu_{k}\right|
$$

and by $[3$, Theorem 1],

$$
\left|\mu_{k N}-\mu_{k}\right|=O\left(\sigma_{N}\right), \quad \text { where } \sigma_{m} \equiv \max _{I \times I}\left|\eta_{m}(x, t)\right|
$$

therefore, it remains to prove that

$$
\tilde{\mu}_{k N}-\mu_{k N}=O\left(\sigma_{n}^{2}+\left|\widetilde{y}_{k}^{(n)}-y_{k}^{(n)}\right|_{n}^{2}+\left(\tilde{\mu}_{k n}-\mu_{k n}\right)^{2}\right),
$$

for which it will be shown below that

$$
\left|\widetilde{y}_{k}^{(N)}-y_{k}^{(N)}\right|_{N}=O\left(\sigma_{n}+\left|\tilde{y}_{k}^{(n)}-y_{k}^{(n)}\right|_{n}+\left|\tilde{\mu}_{k n}-\mu_{k n}\right|\right) .
$$

The error estimate for $\tilde{y}_{k N}(x)$ will be deduced from

$$
\left|\tilde{y}_{k N}(x)-y_{k}(x)\right| \leqslant\left|\tilde{y}_{k N}(x)-\tilde{\mu}_{k N}^{-1} \mu_{k N} y_{k N}(x)\right|+\left|\tilde{\mu}_{k N}^{-1}\left(\mu_{k N}-\tilde{\mu}_{k N}\right) y_{k N}(x)\right|
$$

$$
+\left|y_{k N}(x)-y_{k}(x)\right| \text {. }
$$

To bound $\left|\tilde{\mu}_{k N}-\mu_{k N}\right|$, observe that for sufficiently large $n$ and $N$ it follows by (6) and [3, Theorem 2] that $\mu_{k N}$ is a well-separated eigenvalue of $K^{(N)}$ such that

$$
\left|\tilde{\mu}_{k N}-\mu_{k N}\right|=\min _{i}\left|\tilde{\mu}_{k N}-\mu_{i N}\right|,
$$

which by Theorem 2 implies

$$
\left|\tilde{\mu}_{k N}-\mu_{k N}\right| \leqslant \epsilon_{k N} \equiv\left|H \widetilde{z_{k}}-\widetilde{\mu}_{k N} \widetilde{z}_{k}\right|=\left|K^{(N)} \widetilde{y}_{k}^{(N)}-\widetilde{\mu}_{k N} \widetilde{y}_{k}^{(N)}\right|_{N}
$$

$$
\text { where } H_{i j} \equiv K\left(x_{i N}, x_{j N}\right) \sqrt{w_{i N} w_{j N}} \text { and } \widetilde{z}_{k i} \equiv y_{k i}^{(N)} \sqrt{w_{i N}}, i, j=1, \ldots, N \text {. }
$$

Further, to obtain a bound for $\left|\widetilde{y}_{k}^{(N)}-y_{k}^{(N)}\right|_{N}$, let $\widetilde{y}_{k}^{(N)}$ have the expansion

$$
\tilde{y}_{k}^{(N)}=\sum_{j=1}^{N} c_{j} y_{j}^{(N)}, \quad c_{k}>0,
$$

with $\left\{y_{j}^{(N)}\right\}$ forming a set satisfying (see Definition (4) and [3, Section 3])

$$
\left(y_{p}^{(N)}, y_{q}^{(N)}\right)_{N}=\delta_{p q}, \quad p, q=1, \ldots, N
$$


then

$$
\left|\tilde{y}_{k}^{(N)}\right|=\sum_{j=1}^{N}\left|c_{j}\right|^{2}=1,
$$

and by a procedure similar to that applied in [6, pp. 172-173]

$$
\left|\widetilde{y}_{k}^{(N)}-y_{k}^{(N)}\right|_{N}^{2} \leqslant \delta_{k}^{(N)}\left(1+\delta_{k}^{(N)}\right), \quad\left|\tilde{\mu}_{k N}-\mu_{k N}\right| \leqslant d_{k N} \delta_{k}^{(N)}\left(1-\delta_{k}^{(N)}\right)^{-1},
$$

$$
\text { where } d_{k N} \equiv \min _{j \neq k}\left|\widetilde{\mu}_{k N}-\mu_{j N}\right| \text { and } \delta_{k}^{(N)} \equiv \epsilon_{k N}^{2} d_{k N}^{-2} \quad \text { with } \epsilon_{k N} \text { defined in (9), }
$$

provided that $\delta_{k}^{(N)}<1$. To establish (7), observe that

where

$$
\tilde{y}_{k i}^{(N)}-y_{k i}^{(N)}=d_{i}^{(1)}+d_{i}^{(2)}+d_{i}^{(3)}
$$

$d_{i}^{(1)} \equiv \tilde{y}_{k i}^{(N)}-y_{k n}\left(x_{i N}\right), \quad d_{i}^{(2)} \equiv y_{k n}\left(x_{i N}\right)-y_{k}\left(x_{i N}\right), \quad d_{i}^{(3)} \equiv y_{k}\left(x_{i N}\right)-y_{k N}\left(x_{i N}\right)$

Now

$$
\begin{aligned}
& \tilde{y}_{k i}^{(N)}=\left[\sigma_{N}^{*}\left(\tilde{y}_{k n}\right)\right]^{-1} \tilde{y}_{k n}\left(x_{i N}\right), \quad \text { where } \sigma_{N}^{*}(u) \equiv\left[\sum_{i=1}^{N} w_{i N}\left|u\left(x_{i N}\right)\right|^{2}\right]^{1 / 2}, \\
& \tilde{y}_{k n}\left(x_{i N}\right)-y_{k n}\left(x_{i N}\right)=\mu_{k n}^{-1} \tilde{\mu}_{k n}^{-1}\left(\mu_{k n}-\tilde{\mu}_{k n}\right) \tilde{y}_{k n}\left(x_{i N}\right) \\
& +\mu_{k n}^{-1} \sum_{j=1}^{n} w_{j n}\left[\widetilde{y}_{k j}^{(n)}-y_{k j}^{(n)}\right] K\left(x_{i N}, x_{j n}\right), \\
& \sigma_{N}^{*}\left(y_{k n}\right)=\left[1+\mu_{k n}^{-2} \sum_{p, q=1}^{n} w_{p n} w_{q n}\left[\eta_{N}\left(x_{p n}, x_{q n}\right)-\eta_{n}\left(x_{p n}, x_{q n}\right)\right] y_{k q}^{(n)} \overline{y_{k p}^{(n)}}\right]^{1 / 2}, \\
& \left\|y_{k m}\right\|^{2}=1-\mu_{k m}^{-2} \sum_{i, j=1}^{m} w_{i m} w_{j m} \eta_{m}\left(x_{i m}, x_{j m}\right) y_{k j}^{(n)} \overline{y_{k i}^{(n)}} .
\end{aligned}
$$

Hence

$$
\begin{aligned}
\left|\sigma_{N}^{*}\left(\widetilde{y}_{k n}\right)-1\right| & \leqslant\left|\sigma_{N}^{*}\left(\widetilde{y}_{k n}\right)-\sigma_{N}^{*}\left(y_{k n}\right)\right|+\left|\sigma_{N}^{*}\left(y_{k n}\right)-1\right| \\
& \leqslant\left|\sigma_{N}^{*}\left(\widetilde{y}_{k n}-y_{k n}\right)\right|+\left|\sigma_{N}^{*}\left(y_{k n}\right)-1\right| \\
& =O\left(\left|\widetilde{\mu}_{k n}-\mu_{k n}\right|\right)+O\left(\left|\widetilde{y}_{k}^{(n)}-y_{k}^{(n)}\right|_{n}\right)+O\left(\sigma_{N}\right)+O\left(\sigma_{n}\right), \\
d_{i}^{(1)} & =O\left(\left|\widetilde{y}_{k}^{(n)}-y_{k}^{(n)}\right|_{n}+\left|\tilde{\mu}_{k n}-\mu_{k n}\right|+\sigma_{n}\right)
\end{aligned}
$$

and by [1, Theorem 3], [1, Eq. (10)] and (11),

$$
d_{i}^{(2)}=O\left(\sigma_{n}\right), \quad d_{i}^{(3)}=O\left(\sigma_{N}\right)
$$

which establishes (7).

For the sake of error estimation of $\widetilde{y}_{k N}(x)$, apply the Cauchy-Schwarz inequality to the first two terms at the right-hand of (8) to obtain 


$$
\begin{aligned}
\mid \widetilde{y}_{k N}(x)- & \tilde{\mu}_{k N}^{-1} \mu_{k N} y_{k N}(x)|+| \widetilde{\mu}_{k N}^{-1}\left(\mu_{k N}-\tilde{\mu}_{k N}\right) y_{k N}(x) \mid \\
& \leqslant\left|\widetilde{\mu}_{k N}^{-1}\right|\left[\widetilde{y}_{k}^{(N)}-\left.y_{k}^{(N)}\right|_{N}+\left|\mu_{k N}^{-1}\left(\widetilde{\mu}_{k N}-\mu_{k N}\right)\right|\right] \sqrt{G_{N}(x)}
\end{aligned}
$$

where

$$
G_{N}(x) \equiv \sum_{j=1}^{N} w_{j N}\left|K\left(x, x_{j N}\right)\right|^{2},
$$

with bounds for $\left|\tilde{y}_{k}^{(N)}-y_{k}^{(N)}\right|_{N},\left|\tilde{\mu}_{k N}-\mu_{k N}\right|$ and $\mu_{k N}$ obtained from (10); the bound for the last term of the right-hand of $(8)$ is obtained by application of $[3$, Theorem 3] or of the remark in [3, Section 4] with

$$
\left|y_{k j}^{(N)}\right| \leqslant\left|\widetilde{y}_{k j}^{(N)}\right|+\left|\widetilde{y}_{k j}^{(N)}-y_{k j}^{(N)}\right| \leqslant\left|\widetilde{y}_{k j}^{(N)}\right|+w_{j N}^{-1 / 2}\left|\widetilde{y}_{k}^{(N)}-y_{k}^{(N)}\right|_{N}
$$

and $\left|\widetilde{y}_{k}^{(N)}-y_{k}^{(N)}\right|_{N}$ bounded by (10).

3. Numerical Results. To illustrate the superiority of the new numerical solution and of the new error estimates, the first example presented in [3, Section 2] is taken for comparison. The error estimates for the $\widetilde{\mu}_{k N}$ presented in the following table are those obtained, using the triangle inequality in (5), with the estimate (10) for $\mid \widetilde{\mu}_{k N}-$ $\mu_{k N} \mid$. The bounds for $\left|\mu_{k N}-\mu_{k}\right|$ and the $\mu_{j N}$ and the best error estimate for $\mu_{k n}$ are obtained in [3, Section 5], using the fact that $\mu_{k}$ is the nearest characteristic value to $\mu_{k n}$ and $\mu_{k N}$, and

$$
\eta_{m}(x, t)=\frac{1}{6(m-1)^{2}} \begin{cases}3 t A_{m}(x) B_{m}(x)+F_{m}(t), & x \leqslant t, \\ 3 x A_{m}(t) B_{m}(t)+F_{m}(x), & x \geqslant t,\end{cases}
$$

\begin{tabular}{|c|c|c|c|c|c|c|}
\hline Case & $k$ & $\begin{array}{l}\text { Best error } \\
\text { estimate for } \\
\mu_{k n} \text { by [3] }\end{array}$ & $\begin{array}{l}\text { Error } \\
\text { estimate } \\
\text { for } \widetilde{\mu}_{k N}\end{array}$ & $\begin{array}{l}\text { Actual } \\
\text { error } \\
\text { for } \widetilde{\mu}_{k N} \\
\end{array}$ & $\begin{array}{l}\text { Error } \\
\text { estimate } \\
\text { for } \widetilde{y}_{k N}(x)\end{array}$ & $\begin{array}{l}\text { Actual maxi- } \\
\text { mal error for } \\
\widetilde{y}_{k N}(l / m), \\
l=0,1, \ldots, m\end{array}$ \\
\hline$n=101$ & 1 & $9.074 \cdot 10^{-5}$ & $9.126 \cdot 10^{-7}$ & $1.25 \cdot 10^{-7}$ & 0.001552 & $1.75 \cdot 10^{-4}$ \\
\hline$N=1000$ & 2 & $4.342 \cdot 10^{-4}$ & $5.046 \cdot 10^{-6}$ & $9.11 \cdot 10^{-8}$ & 0.0745 & $9.167 \cdot 10^{-4}$ \\
\hline$m=3000$ & 3 & $1.006 \cdot 10^{-3}$ & $1.137 \cdot 10^{-5}$ & $8.53 \cdot 10^{-8}$ & 0.721 & $2.15 \cdot 10^{-3}$ \\
\hline$n=201$ & 1 & $2.27 \cdot 10^{-5}$ & $2.274 \cdot 10^{-7}$ & $3.14 \cdot 10^{-8}$ & 0.0004524 & $5.177 \cdot 10^{-5}$ \\
\hline$N=2001$ & 2 & $1.09 \cdot 10^{-4}$ & $1.26 \cdot 10^{-6}$ & $2.3 \cdot 10^{-8}$ & 0.0163 & $2.085 \cdot 10^{-4}$ \\
\hline$m=6000$ & 3 & $2.52 \cdot 10^{-4}$ & $2.9 \cdot 10^{-6}$ & $2.167 \cdot 10^{-8}$ & 0.165 & $5.652 \cdot 10^{-4}$ \\
\hline
\end{tabular}

where (the formula for $F_{n}$ in [3, Section 2, Example 1] is in error)

$$
\begin{aligned}
A_{m}(z) \equiv(m-1) z-[(m-1) z], B_{m}(z) \equiv 1-A_{m}(z), \\
F_{m}(z) \equiv 1-z+A_{m}(z) B_{m}(z)\left(3 z-\frac{2 A_{m}(z)-1}{m-1}\right) .
\end{aligned}
$$

The numerical solution for $\widetilde{y}_{k N}(x)$ is the function defined by (8) generated by the approximate eigenvector $\tilde{y}_{k}^{(n)}$ corresponding to the $k$ th negative eigenvalue $\mu_{k n}$. 
Department of Mathematics

Technion, Israel Institute of Technology

Haifa, Israel

1. P. LINZ, "On the numerical computation of eigenvalues and eigenvectors of symmetric integral equations," Math. Comp., v. 24, 1970, pp. 905-910. MR 43 \#1461.

2. S. G. MIHLIN, Lectures on Linear Integral Equations, Fizmatgiz, Moscow, 1959; English transl., Russian Monographs and Texts on Advanced Math. and Phys., vol. 2, Gordon and Breach, New York; Hindustan, Delhi, 1960. MR 23 \#A490; 24 \#A3483.

3. E. RAKOTCH, "Numerical solution for eigenvalues and eigenfunctions of a Hermitian kernel and an error estimate," Math. Comp., v. 29, 1975, pp. 794-805. MR 51 \#956.

4. H. WIELANDT, Error Bounds for Eigenvalues of Symmetric Integral Equations, Proc. Sympos. Appl. Math., Vol. 6, Amer. Math. Soc., Providence, R. I.,1956, pp. 261-282. MR 19, 179.

5. J. H. WILKINSON, Rounding Errors in Algebraic Processes, Prentice-Hall, Englewood Cliffs, N. J., 1963. MR 28 \#4661.

6. J. H. WILKINSON, The Algebraic Eigenvalue Problem, Clarendon Press, Oxford, 1965. MR 32 \#1894. 